\title{
Rythmes circadiens : leurs bases anatomiques, fonctionnelles et moléculaires
}

\section{Gérard Mick Michel Jouvet}

\footnotetext{
CRE : cyclic AMP responsive element CREB : (yclic AMP responsive element binding protein

CREM : cyclic AMP responsive element modulator

ERK : extracellulary-regulated kinase GRP : gastrin releasing peptide.

mGluR : récepteur métabotropique du glutamate.

NMDA : N-méthyl-I)-aspartate

NFG-IA : nerve growth factor-IA

PHI : peptide histidine-isoleucine.

RHT : tractus rétino-hypothalamique.

SCN : noyau supra optique

SRE : serum responsive element

VIP : vasoactive intestinal peptide
}

Observés chez tous les êtres vivants, les rythmes circadiens sont l'expression des variations cycliques des fonctions biologiques qui suivent une période proche de vingt-quatre heures. Chez les mammifères, la genèse de ces rythmes et leur synchronisation sur le cycle solaire dépendent d'une structure cérébrale, le noyau suprachiasmatique, horloge biologique interne mise à l'heure par une information provenant de la rétine. La régulation des rythmes neuro-endocriniens par la lumière fait aussi intervenir un ensemble de structures nerveuses, cibles d'un contingent de voies rétiniennes indépendantes des voies visuelles, appelées voies photiques. La connaissance de l'organisation anatomofonctionnelle de ce système spécifique peut constituer une base pour la mise au point de traitements des troubles survenant lors du décalage horaire, du travail posté, ou de syndromes pathologiques tels que la dépression saisonnière.

\section{ADRESSE}

(;. Mick : neurologue, doctevur en nenurosciences. M. Jouvet : professeur de médecine expérimentale i l'universile Claude-Bernard de I.yon, directeur de l'unilé 52 de l'Inserm el de l'trre 1195 du Cinrs. Université Claude-Benard, 69373

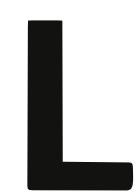

'œeil, organe périphérique de la perception de l'image visuelle, est aussi celui de la perception du temps qui rythme la vie des mammifères. Ce second "sens", à première vue caché, apparaît lorsque l'on examine plus particulièrement le rôle joué par l'environnement lumineux dans la mise en jeu saisonnière de la reproduction. De nombreux rythmes biologiques chez les vertébrés, comme ceux d'activité locomotrice ou des sécrétions hormonales, sont syn- chrones du cycle solaire journalier: ils sont nycthéméraux. Sans information externe, ces rythmes persistent en suivant une période proche mais différente de l'alternance jour-nuit: ils sont circadiens. L 'alternance solaire représente ainsi le synchroniseur naturel de rythmes qui sont endogènes. Une telle anticipation fonctionnelle et son adaptation temporelle à l'environnement, qui concernent au premier chef les fonctions homéostasiques et reproductrices, optimalisent la survie individuelle et de l'es- 
pèce et concourent au bien-être de l'individu.

\section{Une horloge biologique interne mise à l'heure via la rétine}

La structure anatomique responsable de la génération des rythmes circadiens et de leur synchronisation sur le cycle nycthéméral chez les mammifères est un groupe de neurones interconnectés situé à la base du cerveau, le noyau suprachiasmatique de l'hypothalamus (SCN), cible d'une voie nerveuse issue de la rétine, le tractus rétino-hypothalamique (RHT) (figure 1). La lésion du SCN chez les rongeurs s'accompagne d'une disparition de l'organisation circadienne de l'activité locomotrice, de la prise hydrique et des variations de la concentration plasmatique en corti- costérone et en mélatonine, hormone sécrétée par la glande pinéale, impliquée elle-même dans la régulation des rythmes circadiens et saisonniers $[1,2]$. Le rythme d'activité locomotrice est restauré après greffe hétérologue du SCN, et suit alors la période propre du donneur [3]. Une lésion des voies optiques qui épargne le RHT provoque une cécité mais ne perturbe pas l'expression des rythmes, alors que la section du RHT est suivie de la disparition de la synchronisation nycthémérale des rythmes, sans trouble visuel associé [4]. Les neurones rétiniens donnant naissance au RHT ont une morphologie atypique et représentent moins de $0,1 \%$ de l'ensemble des neurones donnant naissance aux voies optiques [5]. Ils sont répartis sur la rétine et distribuent l'information sur le SCN de façon incompatible avec les

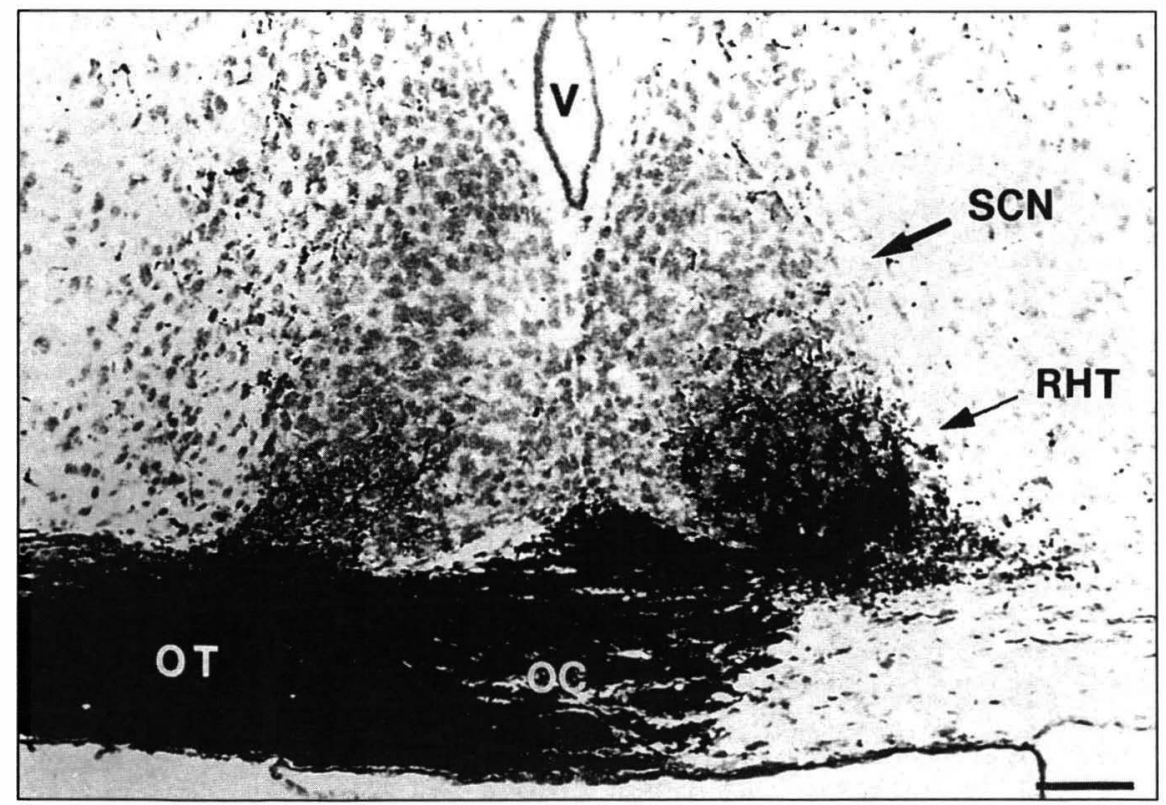

Figure 1. Coupe frontale de la base du cerveau d'un petit mammifère. Le noyau suprachiasmatique (SCN) est identifiable comme un groupe compact de petits neurones colorés par le crésyl violet. Cette structure paire est située de part et d'autre du troisième ventricule (V), cavité liquidienne médiane, et au-dessus du chiasma optique $(O C)$, lieu de croisement des fibres optiques provenant des deux yeux et qui se divise en tractus optiques (OT) destinés aux hémisphères cérébraux. Le SCN reçoit des informations de la rétine par un contingent spécifique de fibres nerveuses, le tractus rétino-hypothalamique (RHT), qui se distribue principalement dans sa partie ventrale et latérale, dans l'hémisphère cérébral du côté opposé à l'œil dont il est issu. Les fibres optiques et les terminaisons du RHT sont révélées ici par leur contenu opaque en traceur qui, injecté dans l'œil et capté par les neurones rétiniens, a migré le long des fibres de projection de ces neurones. Barre d'échelle: $100 \mu \mathrm{m}$.

$\mathrm{m} / \mathrm{s} n^{\circ} /$, vol. 11, janvier 95 contraintes inhérentes à la fonction visuelle imposant une ségrégation spatiale de l'information [6]. Les neurones du SC.N répondent linéairement aux variations de luminance atteignant une très large portion de la rétine, la lumière verte ayant un maximum d'efficacité, caractéristiques également incompatibles avec la reconnaissance des formes, contrastes et couleurs propre à la vision, mais compatibles avec la détection des variations lentes de l'éclairement ambiant [7]. Chez le hamster, la synchronisation du rythme d'activité locomotrice par la lumière dépend directement du nombre absolu de photons incidents sur la rétine au cours d'une période de plusieurs dizaines de secondes [8].

Une caractéristisque fondamentale du mécanisme synchroniseur est sa dépendance temporelle. Une stimulation lumineuse brève $(1 \mathrm{~min})$ et d'intensité modérée (< 10 lux) en début de période nocturne retarde les rythmes, l'horloge intégrant l'information comme un retard d'apparition de la période nocturne; la stimulation en fin de nuit avance les rythmes [1]. Chez le hamster privé de lumière pendant plusieurs jours, ces resynchronisations ne peuvent avoir lieu qu'au cours de la phase d'activité locomotrice, qui correspond, en condition normale, à la phase nocturne (figure 2). L'horloge est donc alternativement sensible ou insensible à une nouvelle stimulation selon un rythme circadien. La stimulation lumineuse induit une resynchronisation des rythmes dont l'amplitude du décalage de phase par rapport à la synchronisation antérieure varie presque linéairement en fonction de la luminance [1]. Une telle organisation fonctionnelle ressemble à celle d'une bascule électronique à commande photoélectrique, dont la rétine serait à la fois un capteur (photorécepteurs) et un compteur (neurones rétiniens) situés en périphérie, et dont le SCN serait un intégrateur à fenêtre périodique en situation centrale. Une activité comportementale soutenue, provoquée par exemple par un changement inopiné de cage et de roue au cours de la période de repos, peut également jouer le rôle de synchroniseur efficace chez certains individus d'une population de hamsters [9]. 


\section{RÉFÉRENCES}

1. Aschoff J. A survey of biological rhythms. In: Aschoff J, ed. Handbook of behavioral neurobiology: biological rhythms. New York: Plenum Press, $1981: 3-8$.

2. Collin J, Faure J, Falcon J, Voisin P, Brisson $\mathrm{P}$, Mirshahi M. Pinéale et rétine. médecine/sciences $1988 ; 4: 16-26$.

3. Ralph MR, Foster RG, Davis FC, Menaker M. Transplanted suprachiasmatic nucleus determines circadian period. Science 1990 ; 247: 975-8

4. Moore RY. Visual pathways controlling neuro-endocrine function. In: Gual C, Ehling FJG, eds. Progress in neuroendocrinology. Amsterdam : Excerpta Medica, $1974: 490-4$.

5. Pickard GE. Morphological characteristics of retinal ganglion cells projecting to the suprachiasmatic nucleus: a horseradish
peroxydase study. Brain Res $1980 ; 183: 458$ 65.

6. Magnin M, Cooper HM, Mick G. Retinohypothalamic pathway: a breach in the law
of Newton-Müller-Gudden? Brain Res 1989 ; $488: 390-7$.

7. Meijer JH, Rietweld WJ. Neurophysiology of the suprachiasmatic circadian pacemaker in rodents. Physiol Reo $1989 ; 69: 671-707$.

8. Nelson DE, Takahashi JS. Sensitivity and integration in a visual pathway for circadian entrainment in the hamster. J Physiol 1991 ; $439: 115-45$

9. Mrosovski N. Phase response curve for social entrainment. J Comp Physiol A 1988 ; $162: 35-46$.

10. van den Pol AN, Finkbeiner SM, Cornell-Bell AH. Calcium excitability and oscillations in suprachiasmatic neurons and glia in vitro. J Neurosci $1992 ; 12$ : 2648-64.

11. Mick G, Yoshimura K, Kiyama H, Tohyama M. Gene expression of metabotropic glutamate receptor subtypes in neuronal subpopulations of the rat suprachiasmatic nucleus. Neuroscience 1995 (sous presse).

12. Mick G, Maeno H, Kiyama H, Tohyama $M$. Marginal topography of neurons expressing the substance $P$ receptor in the rat suprachiasmatic nucleus. Mol Brain Res 1994 ;

\section{Mécanismes moléculaires et cellulaires de la mise à l'heure de l'horloge biologique}

Le relais de l'information lumineuse synchronisante au niveau des neurones du SCN chez le rongeur nocturne dépend de récepteurs membranaires dont le ligand endogène, libéré par le RHT, est l'acide aminé excitateur glutamate. La stimulation de ces récepteurs post-synaptiques provoque une augmentation de la concentration intraneuronale en calcium [10]. Certains récepteurs, non stimulés par l'agent pharmacologique N-méthyl-I)-aspartate (NMDA), sont des canaux sélectivement perméables aux cations monovalents, dont la stimulation induit une dépolarisation membranaire. Celle-ci provoque la mise en jeu secondaire de récepteurs stimulés par le NMDA, sensibles au voltage, qui sont des canaux sélectivement perméables au calcium. La probable mise en jeu simultanée de récepteurs couplés à la phospholipase C (m(iluR) [11] pourrait être responsable du recrutement cytosolique du calcium stocké dans les sites réservoirs endoplasmiques, observé après application locale de glutamate in vitro [9], ainsi que d'une synthèse de diacylglycérol qui agit comme un activateur de la protéine kinase C (PKC). Cette enzyme joue un rôle dans la potentiation des canaux ioniques par le biais de la phosphorylation de certaines sousunités protéiques composant les récepteurs. En outre, le neurotransmetteur peptidique substance $P$, peutêtre libéré par certaines terminaisons du RHT, induit une resynchronisation des rythmes d'activité électrique neuronale du SCN, in vitro, et agit également sur un récepteur couplé à la phospholipase C (PLC). Une synergie d'action des deux neurotransmetteurs libérés ensemble par les fibres rétiniennes stimulées pourrait avoir un effet d'amplification du signal lumineux dans la population neuronale où leurs récepteurs sont colocalisés [12].

Une augmentation calcique intraneuronale peut induire l'expression du gène de réponse précoce $c$-fos par induction de la phosphorylation du facteur de transcription CREB $\left(\mathrm{m} / \mathrm{s} n^{\circ} 8\right.$, vol. 4, p. 523, n¹1, vol. 9, p. 1275), qui devient promoteur du gène en se liant sur le site CRE (calcium-responsive element). Cette phosphorylation, qui peut être bloquée par un antagoniste du récepteur sensible au NMDA, a été détectée au niveau du SCN dans les minutes qui suivent une stimulation lumineuse [13]. L'expression de c-fos dans la partie du SCN où sont concentrés les terminaisons du RHT et les récepteurs du glutamate a été détectée une vingtaine de minutes après stimulation (figure 3). Elle est bloquée par un antagoniste du récepteur sensible au NMDA, ce blocage s'accompagnant de l'absence de resynchronisation des rythmes [14]. Le gène $c$-fos pouvant être induit par activation d'un autre site régulateur, SRE (serum-responsive. element), lui-même activable par le biais de PKC, une synergie d'action supplémentaire pourrait avoir lieu entre récepteurs sensibles au NMDA et récepteurs couplés à PLC. L'induction de $c$-fos est un phénomène directement lié à la stimulation lumineuse, puisqu'une resynchronisation d'origine comportementale n'est pas accompagnée de l'induction du gène [15].

Les gènes de réponse précoce $c-j u n$, jun $B$, jun $I)$ et NGFI-A sont également induits au niveau du SCN après stimulation lumineuse [16]. Les gènes $c$-fos et jun $B$ ne sont exprimés qu'après stimulation lumineuse, $c$-jun et jun I) étant modérément exprimés tout au long du cycle nycthéméral [17]. Les produits de transcription de ces gènes de réponse précoce sont eux-mêmes des facteurs de transcription se liant à la région promotrice de divers gènes, dont certains de réponse tardive. La liaison s'effectue sous la forme d'un dimère Fos/Jun formé par adhérence réciproque au niveau d'un domaine structural commun, les différentes protéines de la famille Jun induisant des affinités et des effets variables du dimère sur le site APl [18]. A la suite d'une stimulation lumineuse, la quantité de dimères liés à $\mathrm{APl}$ au niveau du SCN augmente considérablement, et est maximale deux heures après [19]. Une induction de l'expression de gènes de réponse tardive au cours du processus de synchronisation résulterait de modifications dans la composition des complexes se liant sur API, 


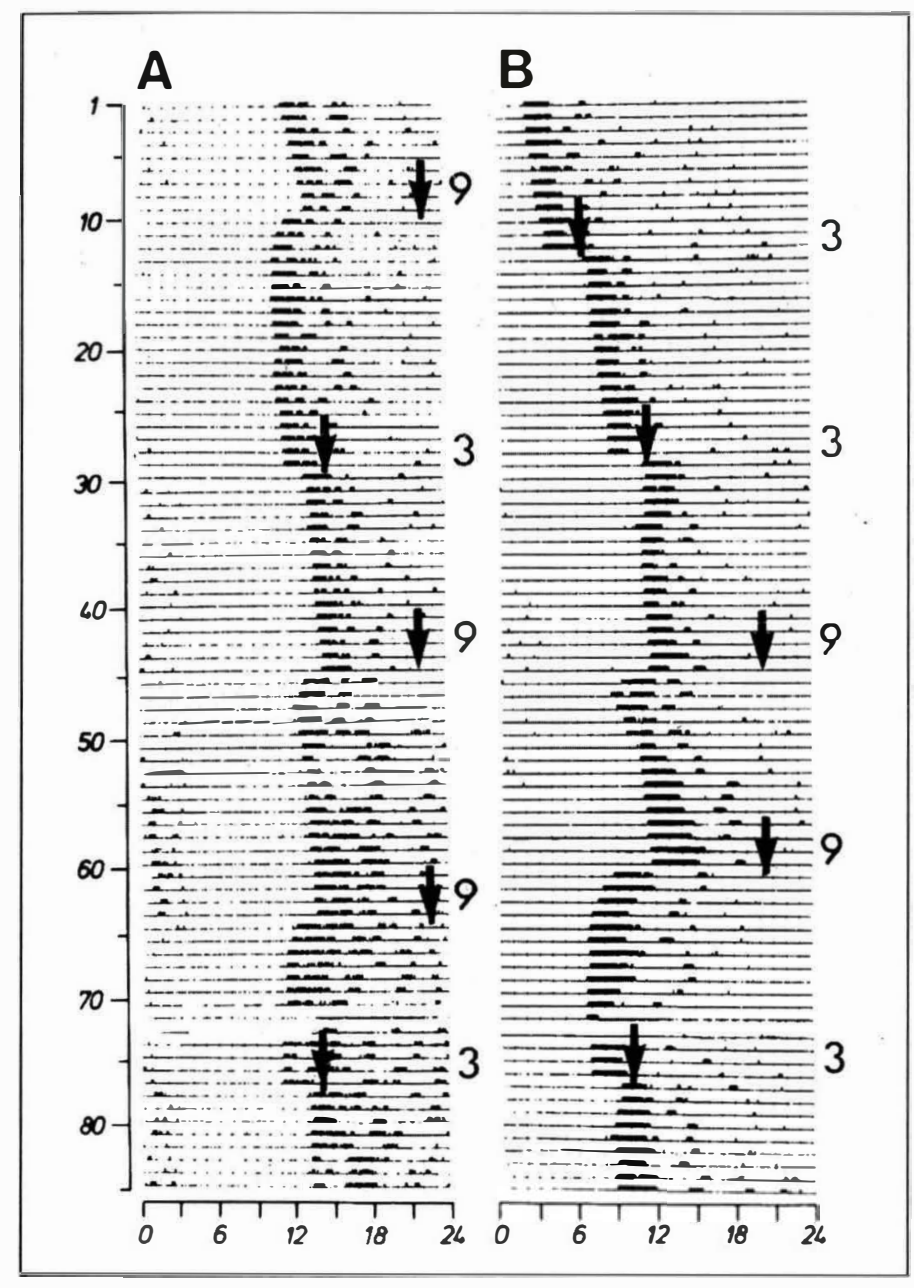

Figure 2. Relevés de l'activité locomotrice de hamsters (A et $B$ ) placés en conditions constantes (lumière rouge de 0,02 lux) pendant plusieurs semaines et recevant un stimulus lumineux de 20 lux pendant 15 minutes en début ou en fin de période d'activité. En abscisse: cycle nycthéméral (jour) de référence de l'observateur, en heures. En ordonnée (de haut en bas): nombre de jours pendant lesquels l'animal est placé en conditions constantes. Les périodes d'activité de l'animal (nuit subjective) sont représentées chaque jour par les barres noires placées sur une ligne $d^{\prime}$ abscisse (actigramme). Dès le premier jour en conditions constantes, le rythme d'activité de l'animal révèle sa nature circadienne: il se décale progressivement du cycle nycthéméral de l'observateur en suivant une période un peu plus longue que 24 heures. Un stimulus lumineux (flèche) délivré 3 heures après le début de la nuit subjective (chiffre 3 en bordure de l'actigramme) retarde le rythme d'environ $1 \mathrm{~h}$ 30. Le stimulus délivré 9 heures après le début de la nuit subjective (chiffre 9) avance le rythme d'environ la même valeur. L'effet de ces stimulations est reproductible chez le même animal et d'un animal à l'autre.

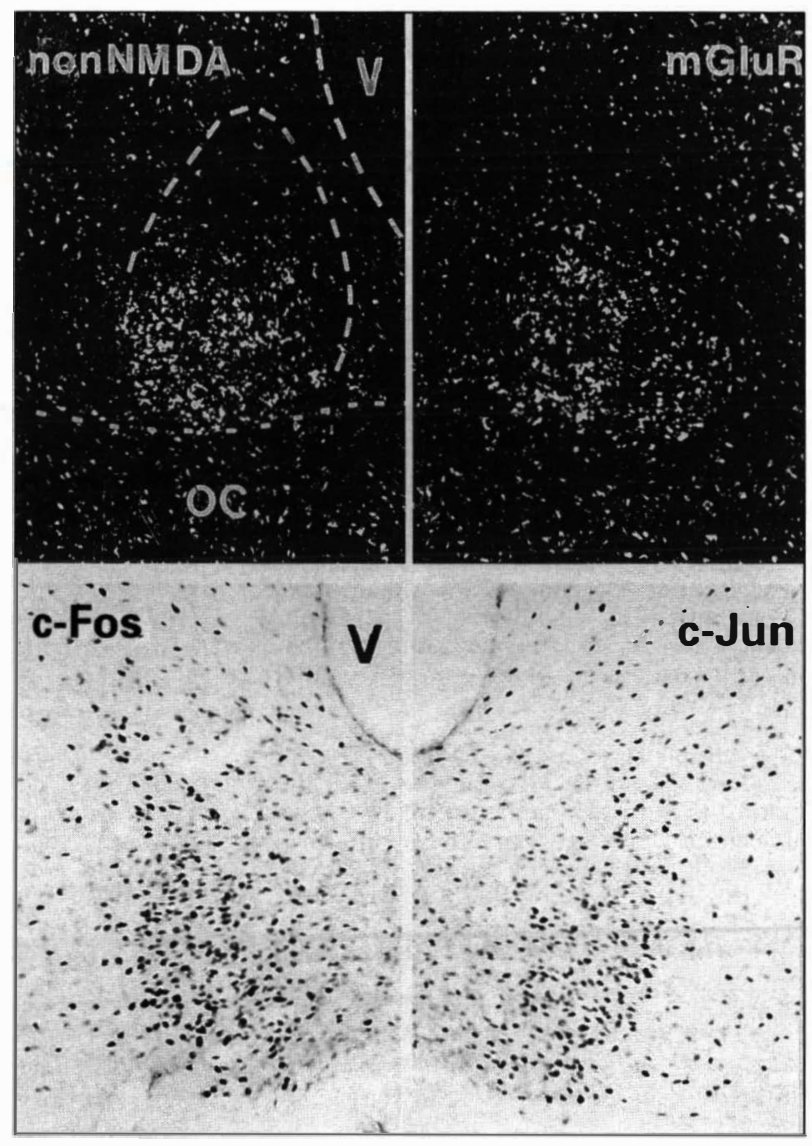

Figure 3. En fond noir: Expression sélective des $A R N$ messagers codant pour des récepteurs de l'acide aminé excitateur glutamate du type non sensible au NMDA (nonNMDA) et du type couplé à l'enzyme phospholipase $C$ (mGluR) au niveau des neurones de la partie ventrale et latérale du noyau suprachiasmatique (SCN) du rat. Le SCN est délimité sur la figure de gauche par un trait discontinu au-dessus du chiasma optique (OC) et au bord du troisième ventricule (V). L'ARN messager codant pour la protéine constituant le récepteur membranaire est mis en évidence par la méthode d'hybridation in situ : les grains $d^{\prime}$ argent qui apparaissent lumineux sur fond noir correspondent au signal produit par une sonde radioactive oligonucléotidique de synthèse, révélée par une émulsion photographique après hybridation avec le segment d'ARN messager dont elle est complémentaire. En fond clair: Expression sélective des gènes de réponse précoce $\mathrm{c}$-fos et jun $\mathrm{B}$ au niveau de la partie ventrale et latérale du SCN de hamster, 2 heures après un stimulus lumineux de 100 lux délivré pendant 15 minutes en début de période nocturne. Les protéines c-Fos et Jun B, localisées dans les noyaux des cellules du SCN, sont révélées par immunohistochimie. Elle ne sont pas détectées dans les structures cérébrales relais des voies visuelles. 


\section{RÉFÉRENCES}

13. Ginty DD, Kornhauser JM, Thompson MA, Bading H, Mayo KE, Takahashi JS, Greenberg ME. Regulation of CREB phosphorylation in the suprachiasmatic nucleus by light and a circadian clock. Srience 1993 ; 260 :238-41.

14. Vindlacheruvu RR, Ebling FJP, Maywood ES, Hastings MH. Blockade of glutamatergic neurotransmission in the suprachiasmatic nucleus prevents cellular and behavioral responses of the circadian system to light. Eur J Neurosei $1992 ; 4: 673-9$.

15. Kornhauser JM, Mayo KE, Takahashi JS Immediate-early gene expression in a mammalian circadian pacemaker: the suprachiasmatic nucleus. In: Young ME, ed. Molecular genetics of biological rhythms. New York: Pergamon Press, 1993 : 271-307.

16. Rusak B, Mac Naughton L, Robertson HA, Hunt SP. Circadian variation in photic regulation of immediate-early gene mRNAs in rat suprachiasmatic nucleus cells. Mol in rat suprachiasmatic nuc
Brain Res $1992 ; 14: 124-30$.

17. Takeuchi Y, Shannon W, Aronin N, Schwartz WJ. Compositional changes of API binding proteins are regulated by light in a mammalian circadian clock. Neuron 1993 ; $11: 825-36$

18. Saint-Arnaud R. Fonction osseuse : fos et les autres. médecine/sciences $1993 ; 9: 1243-6$.

19. Kornhauser JM, Nelson DE, Mayo KE, Takahashi JS. Regulation of jun-B mRNA and AP1 activity by light and a circadian clock. Saience $1992 ; 255$ : 1581-4.

20. Stehle JH, Foulkes NS, Molina CA, Simonneaux $V$, Pévet $P$, Sassone-Corsi $P$. Adrenergic signal direct rhythmic expression of transcriptional repressor CREM in the pineal gland. Nature $1993 ; 365: 314-20$.

21. Sassone-Corsi $\mathrm{P}$. Le gène CREM et les bases moléculaires de l'horloge biologique. médecine/sciences $1993 ; 9: 1253-5$.

22. Chardin P. Protéines Ras et transmission des signaux mitogènes. médecine/sciences $1994 ; 10$ : 657-64

23. Kahn A. La transmission du signal en amont et en aval de Ras. médecine/sciences $1992 ; 8: 1097-9$.

24. Leevers SL, Marshall CJ. MAP kinase regulation - the oncogene connection. Trends
Figure 4. Neuropeptides du noyau suprachiasmatique. Chez le rat, les neurotransmetteurs peptidiques, polypeptide intestinal vasoactif (VIP), peptide histidine-isoleucine (PHI) et peptide de libération de la gastrine (GRP), sont synthétisés dans des populations neuronales localisées sélectivement dans la région ventrale du noyau suprachiasmatique (SCN). Le SCN est délimité en (A) par le trait discontinu en bordure du ventricule $(V)$; $d m$ indique sa partie dorso-médiale. La présence des peptides est révélée par immunohistochimie. Les neurones à GRP sont situés préférentiellement dans la partie ventrale et latérale du SCN alors que les neurones à VIP et PHI, peptides provenant du clivage posttraductionnel du même précurseur, sont localisés dans la partie ventrale et médiane (B). Les deux populations se recouvrent partiellement dans la partie ventrale où certains neurones synthétisent les trois peptides.
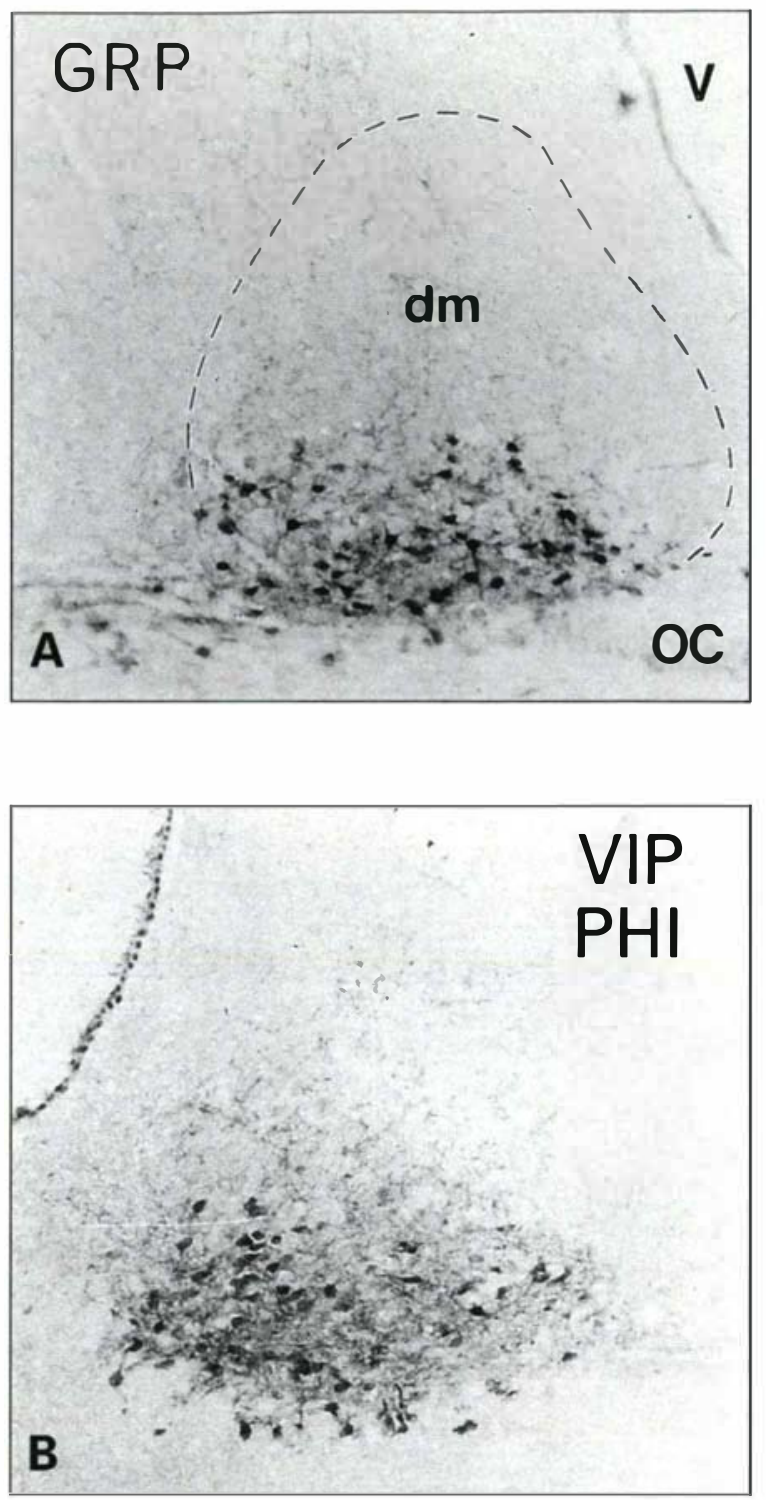

c-Fos et Jun B représentant des signaux spécifiques de la présence de lumière.

La stimulation lumineuse induit également l'expression d'un facteur proche de CREB, mais dont l'activité sur le site CRE est répressive [20, 21$]$. Par ailleurs, des dimères comprenant les protéines de la famille Jun peuvent se lier dans certaines conditions au site CRE. Ces deux mécanismes représentent un rétrocontrole potentiel de l'activation génétique précoce. In vitro, le gène NGFI-A peut être induit par le facteur de croissance nerveux NGF dont le récepteur (NGF$\mathrm{R})$, lorsqu'il est stimulé, active un complexe de type protéine $G$ incluant la protéine Ras. L'activation de cette dernière protéine induit l'activation d'une autre protéine, Raf, qui elle-même peut induire par un phénomène de cascade la phosphorylation de la kinase ERK (extracellularly-regulated kinase) [22] dont l'une des fonctions est d'induire l'expression de gènes de réponse précoce $[23,24]$. NGF-R et ERK ont été détectés dans la partie du SCN où se distribue le RHT. Cette cascade moléculaire pourrait représenter une autre voie d'activation du gène c-fos. Ras et ERK pourraient également intervenir dans l'activation de c-fos par 


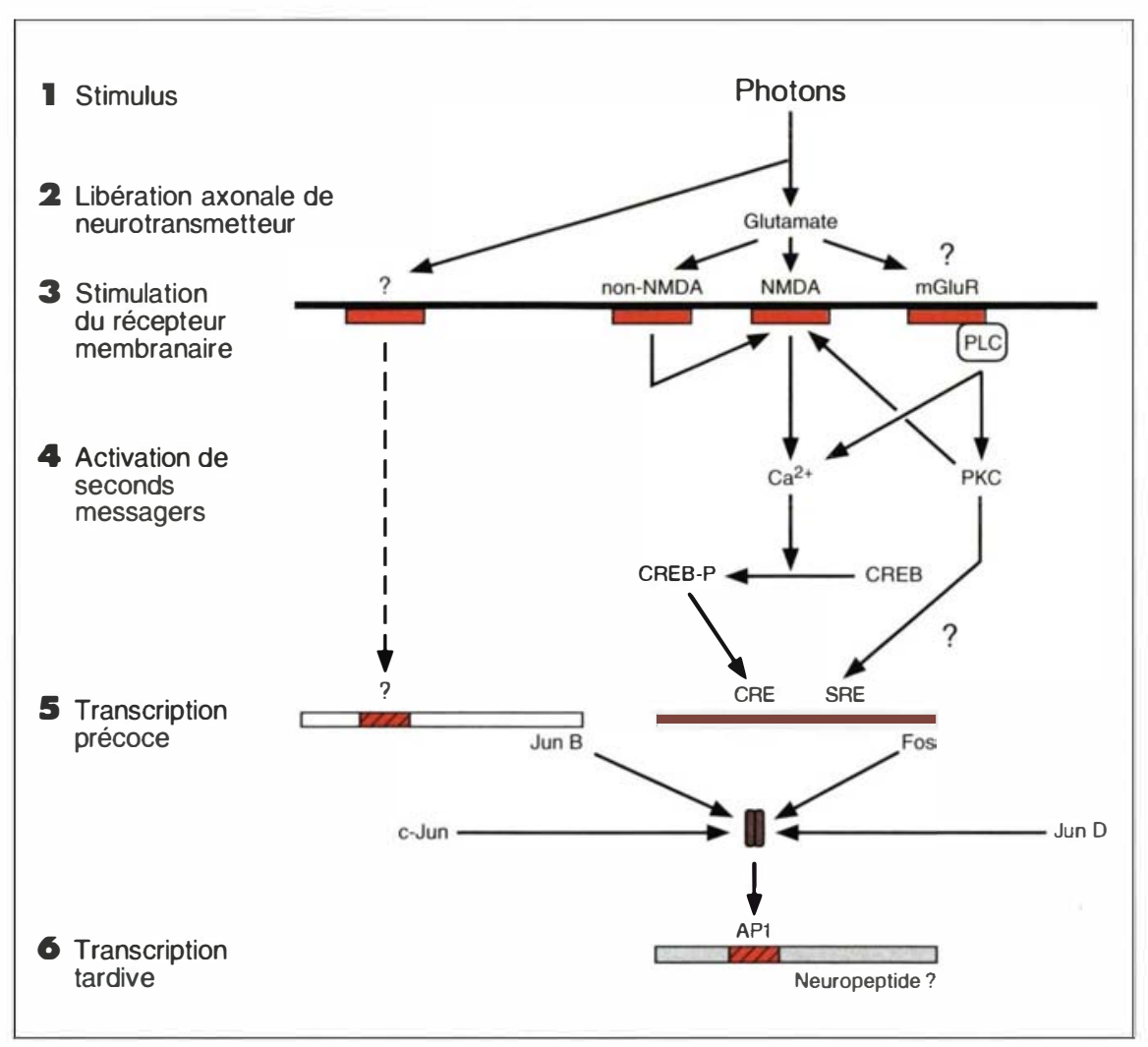

l'intermédiaire de la phosphorylation de CREB.

L'injection au niveau du SCN d'un mélange équimolaire de trois neurotransmetteurs colocalisés dans certains neurones de l'horloge (polypeptide intestinal vasoactif : VIP ; peptide histidine-isoleucine: PHI ; peptide de libération de la gastrine : GRP) (figure 4) resynchronise les rythmes chez le rongeur [25]. Au cours du cycle nycthéméral, l'expression du VIP dans le SCN est minimale durant la phase diurne et maximale durant la phase nocturne, le phénomène inverse étant observé pour le GRP. Après stimulation lumi$\mathrm{m} / \mathrm{s} n^{\circ} \mathrm{I}$, vol. 11 , junvier 95
Figure 5. Une stimulation de la rétine, qui resynchronise les rythmes circadiens chez le hamster, provoque une cascade d'événements moléculaires qui se déroulent à divers niveaux subcellulaires au sein des neurones de la partie ventrale et latérale de I'horloge biologique. La mise en jeu de la protéine c-Fos dans le mécanisme de synchronisation de l'horloge a été récemment étudiée, et ses étapes probables sont décrites ici. La mise en jeu des protéines de la famille Jun dans ce mécanisme n'a pas encore été étudiée. La résultante de la stimulation lumineuse est l'activation tardive d'un gène codant peut-être pour un neuropeptide, par le biais du site AP1 auquel se lient des complexes Fos/Jun dont la composition varie de façon spécifique après stimulation. NMDA = récepteur sensible au N-méthyl-D-aspartate non-NMDA : récepteur insensible au NMDA, mGluR = récepteur du glutamate couplé à la phospholipase C, $P L C=$ phospholipase $C, P K C=$ proté ne kinase $C, C R E B=\mathrm{C}-\mathrm{AMP} / \mathrm{CA}^{2+}$ responsive element binding protein, $C R E=\mathrm{c}-\mathrm{AMP} / \mathrm{Ca}^{2+}$ responsive element, $S R E=$ serum responsive element.

CRE. Le gène codant pour GRP pourrait être la cible d'interactions entre des protéines composant le complexe API et celles de la famille CREB pour l'occupation compétitive du site CRE [27]. Il est possible que certains neurones exprimant GRP soient des interneurones, cibles de la projection rétinienne et exprimant $c$ fos, qui se projettent secondairement sur des neurones coexprimant VIP/PHI et GRP. Enfin, certaines cellules de la glie, qui pourrait jouer un rôle dans les mécanismes de la synchronisation intercellulaire au sein de l'horloge, possèdent des récepteurs du glutamate et expriment $c$-fos. 


\section{RÉFÉRENCES}

25. Albers HE, Liou SY, Stopa EG, Zoeller KT. Interaction of colocalized neuropeptides. Functional significance in the circadian timing system. I Neurosci 1991 ; 11 : $1146-51$.

26. Albers HE, Liou SY, Stopa EG, Zoeller KT. Neurotransmitter colocalization and circadian rhythms. Prog Brain Res 1992 ; 92 : 289-307.

27. Hai T, Curran T. Cross-family dimerization of transcription factors Fos/Jun and ATF/CREB alters DNA binding specificity. Proc Natl Acad Sci USA 1991 ; 88 : 3720-4.

28. Card JP, Whealy ME, Robbins AK, Moore RY, Enquist LW. Two $\alpha$ herpes virus strains are transported differentially in the rodent visual system. Neuron $1991 ; 6: 957$ 69.

29. Ritter S, Dinh TT. Prior optic nerve transection reduces capsaicin-induced degeneration in rat subcortical visual structures. J Comp Neurol 1991 ; 308 : 79-90.

30. Mick G. Description anatomo-fonctionnelle des voies rétinofuges impliquées dans la régulation photique des rythmes biologiques chez les Mammiferes. Définition du système circadien du primate et étude fonctionnelle chez l'homme. Thèse de Doctorat en Neurosciences. Université Claude-Bernard Lyon I, 1991.

31. Cooper HM, Herbin M, Nevo E. Visual system of a naturally microphtalmic mammal: the blind mole rat, Spalax ehrenbergi. $J$ Comp Neurol 1993 ; 328 : 313-50.

32. Foster R, Menaker M. Circadian photoreception in mammals and other vertebrates. In: Light and biological rhythms in man. New York: Pergamon Press, 1993 : 7391.

33. Wever RA. Characteristics of circadian rhythms in human functions. I Neural Trans $1986 ; 21$ (suppl) : 323-73.

34. Moore RY. Human circadian rhythms. In: Moore RY, Klein DC, Reppert SM, eds. Suprachiasmatic nucleus. The mind's clock. New York: Oxford University Press, 1991 : 43043.

35. Reppert SM, Weaver DR, Rivkees SA, Stopa EG. Putative melatonin receptors in a human biological clock. Science $1988 ; 242$ : 78-81.

36. Czeisler CA, Kronauer RE, Allan JS, et al. Bright light induces strong type 0 resetting of the human circadian pacemaker. Science

Quelle que soit l'organisation cellulaire qui sous-tend l'activité du SCN, un élément essentiel du mécanisme de synchronisation et de sa dépendance temporelle est le décours temporel et spatial de la modulation des divers messages transcriptionnels et peptidiques induite par la stimulation lumineuse (figure 5).

\section{Un système spécifique dévolu à l'organisation nycthémérale des fonctions neuro- endocrines}

Des voies nerveuses issues de la rétine et leurs cibles cérébrales, autres que le RHT et le SCN, participent également à la régulation des rythmes circadiens. Des caractéristiques spécifiques communes à ces voies rétiniennes indiquent qu'elles représentent un sous-ensemble spéci- fique de voies optiques. Chez les rongeurs, les aires cérébrales cibles de ces voies sont infectées de façon sélective après injection intra-oculaire d'une souche mutante de virus herpès porcin [28] et dégénèrent après traitement systémique néonatal à la capsaicine [29]. Après stimulation lumineuse entraînant une resynchronisation des rythmes, ces aires cibles expriment également de façon sélective le gène c-fos. Chez les primates, la distribution des voies optiques gagnant ces aires cérébrales est caractéristique, puisqu'elles se projettent préférentiellement sur l'hémisphère cérébral situé du côté de l'œil dont elles sont issues [30]. L'ensemble de ces aires cérébrales cibles, interconnectées, constituent le système circadien. Par extension, on considère aujourd'hui que les structures nerveuses centrales recevant une information de la rétine via des projec-

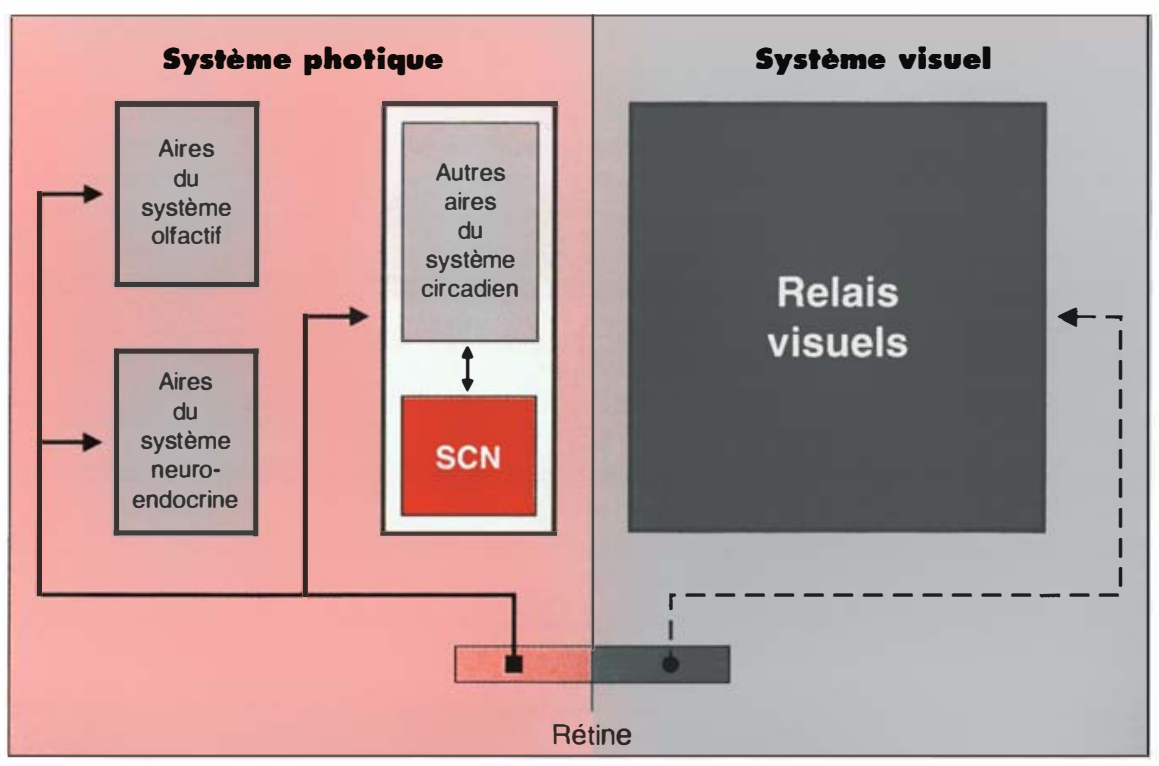

Figure 6. Le système photique et le système visuel constituent deux ensembles anatomiquement et fonctionnellement indépendants, convoyant des informations de nature différente sur des cibles nerveuses centrales distinctes. L'horloge biologique (SCN), représentée en rose, constitue l'élément principal du système circadien, ensemble cible le plus important du système photique. Les autres structures du système circadien, topographiquement proches des relais visuels dans le cerveau, sont interconnectées avec le SCN et participent au contrôle exercé par l'horloge sur les rythmes. Le système photique comprend également des voies beaucoup plus modestes se rendant dans diverses aires de la base du cerveau impliquées dans la régulation des fonctions neuro-endocrines et dans la régulation de la reproduction (aires liées au système olfactif). 


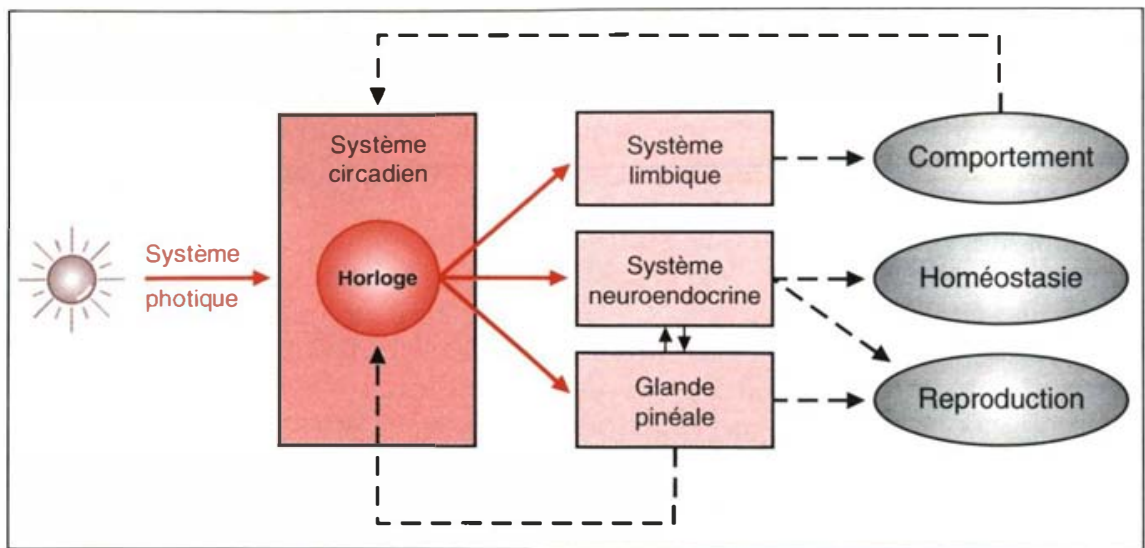

Figure 7. Chez les mammifères, homme inclus, la place du système photique et de sa cible principale, le système circadien, est centrale dans la régulation homéostasique et neuroendocrine ainsi que dans l'organisation rythmique du comportement. Au sein du système circadien, l'activité de l'horloge biologique est modulée par les autres cibles du système photique. Le substratum anatomique sous-tendant la rythmicité des diverses fonctions biologiques est l'ensemble des projections nerveuses de l'horloge sur les divers groupes neuronaux ou neuro-endocrines impliqués spécifiquement dans ces fonctions ou les modulant. Les sorties fonctionnelles de l'organisme, de type comportemental et hormonal, agissent en retour sur le système circadien pour ajuster son activité. Cet ensemble schématique représente la base anatomo-fonctionnelle de l'organisation temporelle des grandes fonctions de l'organisme.

tions modestes récemment décrites et jouant un rôle dans les processus neuro-endocriniens représentent les cibles d'un vaste système projectif de la rétine indépendant du système visuel, appelé système photique, qui est spécifiquement impliqué dans la perception des cycles solaires (figure 6). Une manifestation de cette dualité des voies optiques est l'hypertrophie relative des voies photiques contrastant avec la très sévère régression des voies visuelles chez le rat-taupe d'Israël, mammifère souterrain photopériodique totalement aveugle [31]. Il est très probable que les photorécepteurs rétiniens, dont les messages convergent vers les neurones donnant naissance aux voies photiques, soient, eux aussi, spécifiques. Chez la souris aveugle, porteuse de l'allèle $r d / r d$ responsable de la disparition quasi totale des photorécepteurs rétiniens de type cône et bâtonnet à l'âge adulte, l'entrainement nycthéméral des rythmes persiste et un stimulus lumineux nocturne induit leur resynchronisation ainsi que l'expression de $r$-fos au sein du SCN, d'une façon similaire aux réponses obtenues chez la souris normale [32]. cence neuronale du SCN fréquemment observée chez les personnes âgées et les patients atteints de maladie d'Alzheimer et qui trouve une expression dans l'incidence très élevée des troubles du cycle veille-sommeil chez ces patients. Cependant, malgré de nombreuses similitudes, l'organisation anatomique et fonctionnelle sous-tendant l'existence des rythmes circadiens diffère quelque peu dans l'espèce humaine par rapport à celle des autres mammifères étudiés, les primates supérieurs représentant vraisemblablement une situation intermédiaire. Chez l'homme, les neurones du SCN synthétisent des neurotransmetteurs qui n'ont jamais été mis en évidence dans le SCN d'autres espèces [34]. En situation d'isolement temporel, les rythmes veillesommeil et de température centrale suivent des périodes très différentes et non obligatoirement circadiennes, le seuil de luminance nécessaire pour les synchroniser (2500 lux) étant bien plus élevé que celui qui est habituellement efficace chez les autres espèces diurnes $[33,36]$. Les perturbations de l'organisation cyclique du sommeil ainsi que des rythmes de sécrétions hormonales ne sont pas systématiques chez le non-voyant dont la rétine est totalement lésée. Chez. le primate supérieur, la lésion de l'hypothalamus antérieur abolit dans un premier temps les rythmes de sécrétion de mélatonine et de cortisol, qui réapparaissent ultérieurement et de façon non synchrone [38]. Dans l'espèce humaine, le SCN pourrait agir plutôt comme un chef d'orchestre de plusieurs oscillateurs indépendants, dévolus chacun à une fonction biologique particulière [39]. Le système circadien humain semble en particulier susceptible d'être entraîné très efficacement par les rythmes sociaux d'une façon indépendante du cycle solaire.

Les altérations spécifiques des rythmes circadiens chez l'homme sont des syndromes caractéristiques dont les modes d'expression sont essentiellement des troubles du cycle veille-sommeil ou de l'humeur. Le décalage horaire est typiquement une situation de resynchronisation sur un nouveau cycle solaire et d'activité, imposant à l'horloge interne de se mettre brutalement à une nouvelle heure en entraînant l'ensemble des rythmes. L'origine des troubles est très probablement le conflit initial 


\section{RÉFÉRENCES}

37. Beersma DGM, Daan S. Strong or weak phase resetting by light pulses in humans ? J Biol Rhythms 1993 ; 8: 340-7.

38. Reppert SM, Perlow MJ, Ungerleider L, Mishkin M, Tamarkin L, Orloff DG, Hoffman H, Klein DC. Effects of damage to the suprachiasmatic area of the anterior hypothalamus on daily melatonin and cortisol rhythms in the Rhesus monkey. I Neuroset $1981 ; 1: 141425$.

39. Moore-Ede MC. The circadian timing system in mammals: two pacemakers preside over many secondary oscillators. Fed Proc $1983 ; 42: 2802-8$.

40. Claustrat B, Brun J, David M, Sassolas G, Chazot G. Melatonin and jet-lag: confirmatory result using a simplified protocol. Biol Psychiatry $1992 ; 32$ : 705-11.

41. Akerstedt T. Adjustment of physiological circadian rhythms and the sleep-wake cycle to shift work. In : Monk TH, Folkard S eds. Hours of work. Chichester: John Wiley, 1985 : 185-98.

42. Folkard S, Arendt J, Clark M. Can melatonin improve shift workers' tolerance of the night shift? Some preliminary findings. Chronobiology Int 1993; 10 : 315-20.

43. Dahlitz M, Alvarez B, Vignau J, English J, Arendt J, Parkes JD. Delayed sleep phase syndrome response to melatonin. Lancet $1991 ; 337: 1121-4$.

44. Sack R, Lewy A, Hoban T. Free-running melatonin rhythm in blind people: phase shifts with melatonin and triazolam administration. In : Rensing $L$, van der Heiden $U$, Mackey M, eds. Temporal disorders in human oscillatory systems. Heidelberg: Springer, 1987: 219-24

45. Souètre E, Salvati E, Darcourt G. Chro noendocrinologie de la dépression. médeci ne/sciences 1987 ; 3 : 343-51.

46. Rosenthal NE, Sack DA, Gillin JC, Lewy AJ, Goodwin FK, Davenport Y, Mueller PS Newsome DA, Wehr TA. Seasonal affective disorder: a description of the syndrome and preliminary findings with light therapy. Arch Gen Psychiatry 1984; 41 : 72-80.

47. Mason R, Biello SM. A neurophysiological study of a lithium-sensitive phosphoino-
sitide system in the hamster suprachiasmatic sitide system in the hamster suprachiasmatic
biological clock in vitro. Neurosci Lett 1992 ; $144: 135-8$

48. Chireux M. Pharmacologie des sels de lithium. médecine/sciences $1994 ; 10: 314-7$.
49. Reinberg A. Les rythmes biologiques Paris: Presses Universitaires de France, 1989. transitoire avec la synchronisation antérieure. Il est en effet possible de réduire le délai d'adaptation et l'importance des troubles ; soit en développant une activité physique $\left(\mathrm{m} / \mathrm{s} n^{\circ} 2\right.$, vol. $4, p$. 130) ou en s'exposant à la lumière vive tôt le matin durant les jours qui suivent le changement de fuseau $\left(\mathrm{m} / \mathrm{s} n^{\circ} 7, v o l .5\right.$, p. 520), augmentant ainsi l'amplitude des nouveaux synchroniseurs sociaux et lumineux [36] ; soit par la prise orale de mélatonine un jour avant le changement de fuseau à une heure qui correspond à la fin de soirée dans le nouveau fuseau et en poursuivant cette prise pendant plusieurs jours, simulant ainsi une sécrétion hormonale nocturne, physiologique selon le nouvel horaire, qui resynchronise probablement la sécrétion endogène [40]. En travail posté, particulièrement lors de rotations horaires rapides entrecoupées de périodes de repos prolongé, la sélection préalable des sujets s'adaptant le mieux après une période d'essai s'est avérée efficace [41]. L'habituation du sujet atténue les troubles mineurs qui persistent toujours, et l'exposition à la lumière au cours du travail améliore considérablement le rendement professionnel et la qualité du sommeil diurne. La mélatonine, donnée au moment du coucher, améliore également la qualité du sommeil [42], mais son effet néfaste sur la performance réduit son utilisation thérapeutique lors des périodes de désadaptation du sujet.

Parallèlement à ces altérations fonctionnelles, il existe des syndromes du "dormeur précoce", du "dormeur tardif» et de "désorganisation circadienne du sommeil ", altérations très probablement constitutionnelles pour lesquelles une solution thérapeutique est l'adaptation sociale du sujet à son propre rythme de sommeil. L'exposition à la lumière vive tôt le matin ou la prise de mélatonine en fin de soirée semblent permettre une meilleure adaptation du dormeur tardif lorsqu'un rythme d'activité diurne lui est imposé [43]. Le non-voyant, gêné par un rythme de sommeil totalement désynchronisé des rythmes sociaux, pourrait également bénéficier de la prise de mélatonine au coucher [44]. Le syndrome affectif saisonnier, connu depuis l'Antiquité mais reconnu comme une entité étiopathologique il y a seulement une dizaine d'années, est un syndrome dépressif hivernal récurrent d'incidence supérieure à $5 \%$, d'autant plus fréquent que la latitude est élevée $[45,46]$. Une thérapeutique efficace chez plus de deux tiers des patients est l'exposition à une luminance de 2500 lux pendant deux heures tôt chaque matin, prolongeant ainsi la photopériode en avançant le début de la phase diurne. La physiopathologie de ce syndrome reste inconnue. La psychose maniaco-dépressive, d'expression saisonnière, trouve une thérapeutique préventive de choix dans l'administration chronique de lithium. Cet ion monovalent, parfois efficace dans les algies vasculaires de la face à récurrence saisonnière, allonge la période circadienne chez l'animal en agissant probablement sur le métabolisme neuronal des phosphoinositides $[47,48]$.

On sait à présent que l'efficacité de toute chimiothérapie dépend étroitement de son horaire d'administration, la pharmacocinétique et la pharmacosensibilité tissulaire présentant d'importantes variations nycthémérales [49]. Ces notions récentes ont conduit à une importante amélioration dans la mise au point, l'efficacité et la prévention des effets secondaires de certains traitements, notamment anticancéreux et vasculotropes. Les horloges cellulaires entraînant les fonctions tissulaires sont vraisemblablement synchronisées sur le cycle nycthéméral par des voies humorales sous contrôle de l'horloge interne du système nerveux central. On considère aujourd'hui l'expression périodique d'un processus biologique et sa synchronisation nycthémérale comme une propriété fondamentale et ubiquiste du monde vivant, à tous ses niveaux d'organisation, participant à l'élaboration d'une organisation temporelle de l'organisme (figure 7)

\section{TIRÉS À PART}

G. Mick.

\section{G. Mick.}




\section{Summary}

Circadian rhythms : anatomical, functional and molecular bases

In all living organisms, circadian rhythms are the cyclic expression of biological processes according to a period close to $24 \mathrm{~h}$. The generation of rhythms and their synchronization to the solar cycle in mammals depend upon a central nervous structure, the suprachiasmatic nucleus, an endogenous clock reset by a retinal information. The synchronization mechanism depends on a cascade of molecular events involving various membrane receptors, immediateearly genes and late response genes. The recent discovery of several steps of this cascade in rodents is an outstanding example of direct link between molecular biology and integrative physiology. It has also been established during the last years that in addition to the internal clock, the regulation of neuroendocrine rhythms by light involves several brain areas targets of optic pathways that are independent from the proper visual system: they are called photic pathways. Knowing the organization of this specific system, which is basically similar in humans and other mammals, will provide a basis for the settlement of treatment of dysregulations such as jet lag, shift work or seasonal affective disorders.

\section{Remerciements}

Cette synthèse est inspirée d'études expérimentales et cliniques réalisées à l'Unité 52 et à l'Unité 94 de l'Inserm, à l'hôpital neurologique de Lyon, au département d'anatomie et de neurosciences de l'Université d'Osaka (Japon) et à l'Institut Panum de l'université de Copenhague, avec le soutien de l'Université Claude-Bernard, de la Fédération des Aveugles de France, de la Fondation Fouassier, de la Fondation pour la Recherche Médicale, de la Société Japonaise pour la Promotion de la Science et de la Fondation Takeda. Nos remerciements à J.L. Borach pour la réalisation de l'iconographie. 